\title{
Educación, metodologías, reforma, y el Estado nacional
}

DOI: https://doi.org/10.32870/dse.v0i9.293

\section{Zvi Bekerman*}

Resumen: El presente artículo cuestiona las premisas que históricamente han sostenido la investigación científica y reflexiona, en este sentido, sobre las reformas educativas promovidas por los estados nacionales, las cuales dan cuenta, asimismo, de un sistema económico y político global específico. Es la retórica nacional, a la que todos estamos acostumbrados, la que pone en las escuelas y los profesores la responsabilidad de encontrar "soluciones" a los problemas que perturban a las sociedades modernas. Es precisamente esta construcción retórica la que nos preocupa. El compromiso con la retórica de la reforma a través de la educación dentro de las estructuras del Estado-nación, por lo general, implica abrazar un dilema en el que, si bien no está permitido decir que el cambio no está disponible, la educación es ofrecida en forma universal, libre y obligatoria. Los contextos en los que la educación evoluciona, se establecen de tal manera que no se permite que la educación/escolarización produzca el cambio deseado.

De esta forma, la propuesta analítica conlleva pensar en la educación desde una perspectiva compleja que comienza por desentrañar lo que significa el modelo educativo, y las reformas que se proponen, y pensar en lo que hace falta si reflexionamos desde la perspectiva de los sujetos de contextos sociales específicos que muchas veces no responden a dichos modelos. Este análisis propone volver a representar la ciencia como algo relativo y arbitrario, y tratar de desarrollar nuevas formas de ver los aspectos de las realidades construidas. Palabras clave: Investigación, metodología, educación, reforma y Estado-nación.

\begin{abstract}
This article challenges the assumptions that have historically sustained scientific research and provides the reader an analysis on educational reforms promoted by national states which reflect a specific global economic and political system. The national rhetoric, the one to which we are used, confer to schools and teachers responsibility to find "solutions" to the problems that disturb modern societies. It is precisely this kind of rhetorical construction which should concern us. Committing to the rhetoric of reform through education into the nation-state structures involves embracing a dilemma in which universal, free and compulsory education is provided even when the change is not allowed. The contexts in which education is evolving, are set in a way that does not allow education produce the desired change.

This analysis proposed involves thinking in education from a complex perspective, and leads to reflect on education from a complex perspective, starting to unravel what the educational model is and what reform proposals mean, identifying absences from the perspective of the subjects in specific social contexts that often do not respond to these models. It is proposed here then returning to conceive science as relative and arbitrary, and try to develop new ways to understand the issues of constructed realities. Keywords: Educative Systems, Education Reforms, State-Nation, Science
\end{abstract}

No hay nada nuevo en lo que voy a decir. Biólogos (Mayr, 1988), filósofos (Churchman, 1968, 1979), e incluso simples antropólogos (Bateson, 1979) han reconocido desde hace tiempo, los límites del sueño moderno de la omnisciencia, limitado principalmente por la complejidad de un sistema, que no va a ceder fácilmente a nuestras herramientas científicas tradicionales. A pesar de que todo se ha dicho, al mirar a mi alrededor en la academia, me pregunto si no es necesario re-

* School of Education, Melton Centre, Hebrew University of Jerusalem. Escuela de Educación, Centro Melton, Universidad Hebrea de Jerusalem. 
cordar una vez más lo ya dicho. Nuestras metodologías y herramientas metodológicas son nodales para este compromiso y se sitúan en la base de los paradigmas que guían nuestra investigación.

Guiado por perspectivas positivistas cuantitativas convencionales, cuando reflexiono sobre todos estos temas, podría esperarse de mí que busque nuevas fórmulas para, por ejemplo, mejorar nuestra posibilidad de manipular simultáneamente más variables, o para mejorar las representaciones gráficas de los patrones de relaciones, o, mejor aún, mejorar la investigación de pequeñas muestras. Haciendo esto, reconocería implícitamente que el investigador, dada una buena metodología, es casi irrelevante en la ecuación de la investigación.

Soy un empirista, pero no un positivista. Trabajando como lo hago, en la antropología del aprender y el saber o en la antropología de la educación, estoy convencido desde hace ya mucho tiempo, que las escalas habituales y gráficos producidos por las ciencias sociales ofrecen, en el mejor de los casos, representaciones pobres de mi foco de estudio, que es el aprendizaje de los seres humanos, quienes por el mero hecho de estar vivos, no están finalizados, son intermitentes, están en constante cambio y se niegan obstinadamente a ser reificados. En este sentido, me parece que estoy unido a los biólogos en las antiguas guerras científicas entre las ciencias exactas y las naturales, que cuando criticados por los físicos por su laxo enfoque científico, respondían: ¿Qué más podemos hacer? Lo que estudiamos (nosotros los biólogos) se mueve (Mayr, 1988). Aunque los físicos ya se han sobrepuesto a las perspectivas positivistas simplistas al descubrir que la relatividad, la incertidumbre y el caos rigen en su investigación, los científicos sociales parecen, en su mayor parte, por desgracia, no ser capaces de superar el paradigma positivista tradicional que regía la física antigua.

Podría ser un filósofo "tradicional", que, guiado por el pensamiento occidental moderno que, en pocas palabras, se dice que es un mero "pie de página" a Platón, se dedica a la trayectoria que conecta directamente a Platón con Descartes al intentar, a través de los esfuerzos de un yo solipsista, descubrir la metafísica en la sombra (como en la metáfora platónica). Pero una vez más, la biología viene al rescate y no me preocupo por lo que nunca he visto: la mente o el yo individual, y me adhiero al auténtico empirismo de todos los tiempos, aquel de Darwin, que en 1838 ya sabía que: "El que entiende babuinos haría más por la metafísica que Locke "(Charles Darwin, en su Cuaderno de Notas M, como se cita en Barrett, Gautrey, Herbert, Kohn y Smith, 1989: 539).

Con Darwin observo lo que está vivo y al hacerlo, me he dado cuenta de que lo que se necesita para mejorar nuestra investigación social y salir adelante no es otra metodología ni los esfuerzos de una mente individual, sino seres humanos, investigadores -si es que también nosotros, los investigadores-, somos seres humanos. Nosotros somos los que llevamos y aplicamos las metodologías y el análisis.

Descubrí la antropología como disciplina hace casi 40 años, cuando trabajaba como joven moderador en una organización voluntaria que ofrece seminarios para estudiantes de secundaria. No recuerdo exactamente por qué, pero me acuerdo de mi curiosidad sobre lo que la academia 
tenía para decir acerca de mi actividad educacional en ese momento. Leyendo la investigación educativa cuantitativa tradicional, tuve la sensación que lo que se ofrecía en términos de resultados e interpretaciones no reflejaba en absoluto mi experiencia al trabajar en el campo educacional. Por casualidad cogí el libro de Clifford Geertz, "La interpretación de las culturas" (Geertz, 1973), lo leí y me vi inmediatamente atrapado en un diálogo con la complejidad de la comprensión de la vida. Cuando comparé la complejidad, que recientemente había descubierto, con la causa y el efecto, la manipulación de variables y las generalizaciones, que no reflejaban en absoluto mi actividad en las aulas de clase, las descripciones de Geertz hicieron eco de mis experiencias complejas y ofrecieron una mejor manera de mirar y reflexionar sobre lo que mis colegas y yo estábamos haciendo en materia educativa. Desde entonces he estado tratando de entrenar mis sentidos para encontrar y conocer el mundo, junto con pensar en lo que encuentro en él a través de las herramientas tradicionales y las perspectivas paradigmáticas que la antropología ofrece. (Acá entre nosotros, desde ese entonces he abandonado mucho de lo que me gustó en Geertz.)

Hace quince años, empecé a enseñar cursos de metodología cualitativa en la universidad, es decir, empecé a fallar en enseñarles a otros lo que había sido tan útil para mí, y más aún, fallar en el hecho de enganchar a mis alumnos (que hoy en día se han convertido en muchos de mis lectores) en un diálogo acerca de las complejidades que nos hacen humanos, es decir, nuestro deseo insaciable — de hecho nuestra necesidad - de aprender. Quisiera aceptar toda la responsabilidad por mi fracaso. Creo que mis habilidades como maestro podrían llevar toda la culpa, pero creo que la responsabilidad, al menos en parte, está en los muchos años de entrenamiento que los estudiantes y lectores han sufrido en las instituciones creadas por el Estado soberano, que les ha inculcado una ideología occidental moderna, apoyada en un paradigma positivista monológico que justifica su control presente y su supervivencia futura.

Más tiempo (Tiempo: el dios de los exámenes escolares y por ende, el dios del éxito escolar -Chronos-el dios- no sabía qué importante iba a ser, a pesar de haber comido niños, hace ya mucho tiempo atrás); más tiempo permitiría una explicación del desarrollo y la adopción de estas técnicas de investigación basadas en sus intrincadas conexiones y su evolución histórica, fuertemente relacionadas con las nuevas condiciones políticas / económicas de una modernidad que ingresó a Europa. Pero más tiempo no hay. Sólo para dar un ejemplo, por favor recuerden que la primera mención conocida de la palabra 'estadística' aparece en el título de la obra satírica "Microscopium Statisticum", de Heleno Politanus en 1672, probablemente dando sentido de pertenencia a los estatistas o al arte de gobernar. El primer uso del adjetivo en algo parecido a su significado actual se encuentra en el "Statisticum Collegium", en latín moderno, usado por Martin Schmeizel (fallecido en 1747) para un curso de conferencias sobre las constituciones, recursos y políticas de los distintos estados nacionales del mundo. Cuando a esto se suman las conexiones más tristes que existen mostradas por los historiadores, entre las premisas de la psicología (disciplina principal de la investigación educativa en la actualidad) y el desarrollo de los poderes centralistas del Estado y sus economías, 
debemos preguntar, qué podrían significar exactamente estas conexiones. En otras palabras, ¿cómo pueden las ciencias sociales, apoyadas por tales supuestos, servir a intereses políticos particulares? El positivismo social y la política del Estado-nación se llevan bien, muy bien.

Un paralelo puede ser dibujado con la hipótesis de Sapir-Whorf(Sapir, 1921), quien le atribuye un papel importante a la lengua en la formación de nuestros pensamientos. Del mismo modo, el nacionalismo parece dar forma y dirigir nuestras concepciones paradigmáticas más básicas, tanto de la sociedad como de la identidad individual. Si como nos dicen teoréticos críticos hoy, las ciencias sociales sufren de nacionalismo metodológico (Beck, 2011), los recursos y las prácticas discursivas que ofrecen, expresan y refuerzan su poder. Estos recursos apuntan a esquemas culturales muy arraigados que organizan la forma en que interpretamos nuestros entornos en la comunicación verbal. Los patrones de conversación que están disponibles para organizar los procesos comunicacionales de determinados grupos a través de una forma textual mediadora integrada en el ámbito simbólico de un entorno social determinado, son en nuestro caso, patrones determinados por el poder del Estado-nación a través de la constitución actual del campo político a través de dos polos irreductibles de determinación esencialista, por ejemplo: bueno/malo, particular/universal, judío/ árabe, etc. (Neuman y Bekerman, 2001).

Sin embargo, no quiero hablar de quién lleva o comparte la responsabilidad de mi fracaso, lo que quiero hacer es intentar, por lo menos parcialmente, señalar lo que es posible, lo que se desea conseguir cuando se adopta un enfoque etnográfico en la investigación.

Muchos de los consumidores de la investigación educacional, los verdaderos herederos de la superficialidad de las ciencias sociales positivistas, quieren entender una realidad verdadera, esperan encontrar una descripción exacta, y esto sólo en el mejor de los casos, para ayudar a cambiar para bien la presente realidad. Parecen creer que una buena investigación debe ser capaz de ofrecer un curso de acción claro, una fórmula sencilla que, si se sigue, promete el éxito en lo que se ha mostrado insuficiente o fracasado.

Si están involucrados en la política (policy) educativa, creen que necesitan primero ser capaces de ofrecer un análisis convincente y racional de la realidad, y más tarde, algunas conclusiones claras y bien definidas en cuanto a la forma de actuar para cambiar la realidad descrita. El hecho que la educación y su éxito o fracaso no ha cambiado, a pesar de la gran cantidad de investigaciones llevadas a cabo a través de los años, parece no molestarles en absoluto (piensen un momento cuántos hambrientos se podrían alimentar con lo que se ha invertido en investigación educacional). Al reconocer el fracaso de la investigación, dirán que se debe a que la investigación, aún, no se ha hecho correctamente, o en el peor de los casos, debido a que sus recomendaciones no han sido aplicadas correctamente por los maestros, por quienes planean los currículos, por los directores o por los encargados de formular las políticas educativas, etc. Por su parte, muchos de los educadores positivistas, están en busca de soluciones directas, lo teórico les cansa, no tienen tiempo; reaccionan a la teoría como si supieran las raíces históricas de la palabra (del griego theoros), que designa 
a los empleados que fueron autorizados por el soberano para determinar si algo había ocurrido o no, para dar testimonio. Ellos temen a la teoría, como si supieran que su etimología indica su estricta relación con el poder del Estado.

Cuando, por la falta de otras opciones, estos mismos positivistas revisan investigaciones guiadas por métodos etnográficos, todavía esperan descubrir relaciones causales; y se niegan a reconocer que el mundo de la actividad humana es complejo, cambiante, y está siempre influenciado por contextos y trayectorias históricas. Insisten en que, aunque la investigación etnográfica puede ser válida, al dar cuenta de las complejidades de la interacción humana, y al hacer hincapié en los múltiples niveles de análisis contextuales que deben tenerse en cuenta (el micro, mezzo-, macro) todo esto hace frívolo el esfuerzo de investigación y niega la posibilidad de tomar decisiones claras sobre qué hacer para cambiar la situación. Parece que creen que es mucho más humano juzgar, de acuerdo a lo que el observador no tiene disponible lo que los sujetos piensan. Como nuestros peores enemigos, se interesan más en nuestras intenciones que en nuestros actos (vieron alguna vez una intención?). A veces temo que ellos son los verdaderos herederos (con suerte no intencionales -intención, que rara palabra) de una psicologizada y esencializada cosmovisión cuyas relaciones con el desarrollo del Estado-nación parecen ser desconocidas para ellos (Foucault, 1969; 1973).

Participar en un diálogo crítico con estas perspectivas no es tarea fácil. Se trata sobre todo, de volver a representar a la ciencia como algo relativo y arbitrario, y de tratar de desarrollar nuevas formas de ver los aspectos de las realidades construidas. La comprensión humana no es mera representación lingüística, matemática, visual o auditiva, la comprensión es el ejercicio de la proficiencia. Entendemos algo cuando sabemos cómo interactuar con él y usarlo bien. A pesar que tradicionalmente se nos ha enseñado que la ciencia se debe a la formulación de hipótesis y experimentos diseñados para desacreditarla, la formulación de Popper parece insuficiente para lo que está vivo y por lo tanto es complejamente impredecible, ya sea el caso de la célula de un biólogo o de un evento social. Sólo el miedo a la reproducción de las dicotomías, de cuya promulgación acusé al Estado nacional, me impide traer el "todo vale, todo va, todo es relativo" de Feyerbend como argumento de la discusión.

La lucha contra estas perspectivas es llegar a apreciar que el primer paso para comprender, es comprender primero la mejor manera de interactuar con la información que hemos recibido, y que entender es sostener una creativa, agradable, o útil interacción con la información que tenemos al alcance de la mano. Esta interacción, es la creación de significado.

Como Conant postula, la ciencia es una serie interconectada de conceptos y esquemas conceptuales que se han desarrollado como resultado de la experimentación y la observación y son sólo fructíferos para la experimentación y la observación (Conant, 1951). Es así que el proceso implica abandonar la romántica y maldita esperanza de encontrar soluciones rápidas o escribir informes burocráticos loables, diciendo qué cosas -en este caso, programas educativos- trabajan bien o mal. Se trata de reconocer la complejidad de la interacción humana y sus redes, la naturaleza intermi- 
tente de la creación de sentido, y la necesaria exuberancia y deficiencia de la traducción (Ortega y Gasset, 1957). Se trata de utilizar las complejidades reveladas como una palanca para hacer humildes nuestros puntos de vista cuando confrontamos las múltiples facetas de la "realidad". Finalmente, requiere que todos entiendan que la búsqueda antropológica es una, y que para ser aceptable necesita no sólo de un cambio epistemológico, sino también de uno político (Bekerman, 2006). Un cambio político que, esta vez, impida que la investigación sea reducida a las tradicionales fuerzas esencialistas y/o (como en nuestro caso) a dicotomías teóricas y prácticas, y exija en su lugar un cambio político que implique la contaminación de todas las esferas de actividad, reconociendo que toda la investigación y la práctica de la actividad de la identidad y/o el investigador y las identidades de los sujetos, se constituyen en las relaciones de los unos con los otros.

La política, desgraciadamente, es el marco sistemáticamente escondido en el mundo institucional, en el sistema educativo del Estado; escondido con el fin de capturar y conservar a los interesados en la investigación educativa en el paradigma positivista de la vida. Nosotros, todos, tenemos que trabajar duro para descubrir las prácticas banales que el Estado nacional soberano utiliza para atraparnos en sus marcos culturales/en su semiótica. La tarea es similar a la descrita por Duro para las artes: "La tarea de cualquier discusión sobre los marcos y molduras en las artes visuales es, ante todo, contrarrestar la tendencia del marco a la invisibilidad con respecto a la obra de arte" (Duro, 1996: 1). Esta actividad no es fácil. Derrida, en uno de sus escritos menos oscuros, poéticamente apunta a esta dificultad:

"El parergon [accesorio o marco] se destaca tanto del ergon [el trabajo de arte] como del milieu - la pared, se destaca en primer lugar como una figura sobre un fondo. Pero no se destaca como el trabajo de arte. Este último también se destaca sobre un terreno. El marco parergonal se destaca sobre dos terrenos, pero con respecto a cada uno de estos, se funde en el otro. Con respecto a la obra de arte que puede servir como su suelo, se funde en la pared, y luego, gradualmente, en el contexto general. Con respecto a la pared, que es el contexto general, se funde en el trabajo de arte, que se destaca contra el suelo en general. Siempre hay una forma, en la pared, pero el parergon es una forma que tiene su determinación tradicional, no que se destaca, pero que desaparece, se entierra, se borra a sí mismo, se desvanece en el momento en que despliega su gran energía" (Derrida, 1987: 57).

El Estado soberano es un parergon (o marco) cuando presenta perspectivas paradigmáticas en las ciencias sociales. Ellos, el Estado y las ciencias sociales positivistas, se constituyen uno a las otras, sin ser absolutamente intrínsecos o extrínsecos uno con las otras.

Desvincular el nudo que los une, superando el paradigma del Estado-nación depende de encontrar la manera de ofrecer al lector, al investigador, las alfabetizaciones necesarias para que lean al mundo, a nuestro mundo o a cualquier otro.

En términos burkeanos, quiero ofrecerles "dramatismo" (Burke, 1969; Burke, 1969): la comprensión de que las relaciones entre la vida y el teatro no son metafóricas, sino reales; la compren- 
sión de los sistemas simbólicos es la clave para la comprensión de la organización social. Esta alfabetización, requiere abundante teoría y ricas facultades descriptivas a fin de descubrir y hacer frente a la complejidad de los sitios y los fenómenos sociales que esperamos que los lectores o investigadores interpreten. Es así que los lectores necesitan familiarizarse con una variedad de disciplinas y discursos:

- El discurso económico, para deliberar sobre la consumición, los productos básicos, sus suministros y gestionamiento.

- El discurso estético, para discutir la arquitectura, la publicidad y la exposición (display).

- El discurso político, para discutir el cuerpo y el incorporamiento, las políticas, la planificación, la disciplina.

- El discurso etnográfico, para encontrar la belleza de las respuestas particulares a todo lo anterior.

- El discurso histórico, para hablar del cambio en la organización, el consumo, la comunidad.

- Los discursos interpretativos, para articular entendimientos entre cada uno de los textos, y su necesaria intertextualidad en la práctica, que en conjunto, crean la cultura de reforma o de otro tipo.

Todos los anteriores son necesarios para leer el mundo y las actividades que lo constituyen, no sólo en el mundo exterior, sino también dentro de las aulas. Esto podría no ser todo lo que se necesita, pero es un comienzo importante, sin el cual, no se puede alcanzar el entendimiento de lo social.

El desarrollo de la educación masiva, a través de la escuela, está estrechamente relacionado con la revolución industrial y el desarrollo del estado nacional. Ambos se encontraban en la necesidad de reclutar a las masas a su servicio; masas con habilidades cognitivas y conductivas básicas, que pudieran servir a las necesidades de la nación-estado y su estructura económica. Así, las escuelas, no son de ninguna manera arenas desinteresadas en las que los conocimientos o habilidades neutrales se transmiten de la mente de los especialistas a sujetos pasivos. En la era moderna, las escuelas han servido como el principal medio por el cual los soberanos han unificado los diferentes grupos locales, que habitan en las áreas que tuvieron éxito en subordinar, bajo una sola bandera, un idioma y una sola narrativa histórica (Cole, 2001).

A pesar que las reformas educativas se produjeron a nivel local en varios puntos a lo largo de la historia, la noción moderna de la reforma en la educación, está vinculada con la difusión de la educación obligatoria. Las reformas educativas no se generalizaron hasta después que la organizada escolarización fue suficientemente sistematizada. Antes, simplemente no había lo que reformar. En el mundo moderno, el crecimiento económico y la expansión de la democracia (cuya definición aún no está totalmente clara) han aumentado el valor de la educación (no se sabe exactamente para quién), y la importancia de garantizar que todos los niños y adultos tengan acceso a una educación de calidad y eficaz. "Calidad" y "eficaz" tampoco son términos claros. Calidad y eficacia pueden 
ser entendidos en referencia a los estudiantes o al sistema. Las reformas educativas modernas están dirigidas cada vez más a mejorar el éxito de la enseñanza y el aprendizaje en las escuelas; y nuevamente no queda claro para quién, si para los estudiantes o para el sistema.

Lo que sí es claro, es que en muchos países los movimientos de reforma educacional están enmarcados en las declaraciones de crisis. La demarcación es interesante, más aún desde el comienzo de PISA en el año 2000. Es interesante porque nos permite reconocer que el estudio fue iniciado por la Organización para la Cooperación y el Desarrollo Económico (OCDE), que es la organización que se encarga de ayudar a los gobiernos a formular políticas eficaces. Lo interesante de estos estudios, es que localizan los intereses presentes de las posibles reformas, no a nivel del individuo, del estudiante, sino a nivel general, a nivel de la toma de decisiones, para la conducción del sistema educativo a nivel nacional. Por lo menos ahora aclaramos lo que indicamos antes y que no estaba tan claro. Al parecer el OCDE se interesa en aumentar el valor de la educación a nivel nacional, es decir, para el beneficio de la nación no del individuo. La pregunta es, si alguna vez han visto a una nación siendo educada? O si consideran que los intereses de la nación y sus políticos son homólogos con los intereses del individuo. Tal vez, a veces lo son, cuando el individuo es el hijo del presidente, o de otra personalidad perteneciente a las élites del país....la cuestión es, si lo son cuando se trata de los marginados.

Elconcepto"Reforma", esunconceptocomplejo.Comosustantivo,eltérminoseutilizaparadescribirloscambiosenla política, laprácticaola organización. Comoverbo, "reformar", refierealos intentos de corregir un problema identificado. En la educación, al parecer, el objetivo de la reforma es realizar una reestructuración profunda, sistémica y sostenida de la educación pública; o al menos eso nos dicen. A lo largo de la historia de la educación pública, la reforma ha sido un medio para la concepción y promulgación de las visiones del bien colectivo, el bien colectivo.

Desde el establecimiento de escuelas comunes, a través de las luchas de John Dewey en defensa de la educación pública como método principal de la reforma social, a las ambiciones de largo alcance de No Child Left Behind, los esfuerzos de reforma han respondido a condiciones de amplia consecuencia que requieren una deliberación centrada en la ética y una acción orientada al futuro. El problema es que los marginados, los pobres, no se sienten centrados en la ética liberal y necesitan soluciones en el presente.

Al parecer los gobiernos que piden reformar la educación, piden asegurar que todos los ciudadanos reciban alfabetización, y a través de ella, un núcleo común de conocimientos, habilidades y valores. En el occidente (y más), estos pedidos de reforma se basan en perspectivas idealistas en las cuales el aprendizaje se concibe como la búsqueda individual de la excelencia. Los estudiantes van a acumular conocimientos y habilidades de alto nivel cognitivo, necesario para llevar una vida moral y socialmente productiva. El éxito del alumno será recompensado por el logro y el mantenimiento de su posición competitiva en las jerarquías sociales, y de acuerdo a su posición social. Los maestros agentes del poder soberano, son los que articulan las metas y modelan a los alumnos 
(o mejor dicho, las mentes de sus alumnos), y los guían en el camino a tomar. Los maestros son aquellos que miden los logros, y que como todos, reciben la misma educación, aquellos que no tienen logros, son considerados fracasados por falta de motivación.

Desde el punto de vista de los maestros, si la reforma es necesaria, ésta es necesaria a nivel de los alumnos, de sus actividades, o del currículo. Del punto de vista del soberano, si se fracasa, son los maestros quienes necesitan ser reformados.

Quiero sugerir que aquello que el soberano llama "fracaso", no es más que un éxito. El éxito del soberano, quien no lo puede aceptar ni expresar, pues políticamente no sería conveniente que él para su propia existencia necesite de un alto porcentaje de fracaso escolar para poder justificar la existencia de un alto número de marginados. Dice a cambio que el sistema escolar fracasa a pesar de él, el soberano, quien desea que tenga éxito. Los responsables del fracaso son los maestros, ellos son los que necesitan la reforma. Si hubiera buenos maestros, (esto es lo que pretende hacernos creer el soberano) la educación sería exitosa, y el mesías llegaría al mundo (dependiendo de la tradición religiosa, llegaría por segunda vez al mundo). El soberano no quiere que tengamos dudas, respecto a que las grandes promesas de la revolución francesa y el iluminismo pueden y deben ser alcanzadas. Todos podemos y seremos iguales. La promesa del gran elíxir de la igualdad, será alcanzada a través de la educación. Una educación de valores, de carácter, de sabiduría, y todo esto conseguido a través del trabajo intelectual, a través del trabajo cognitivo. Sólo malos colegios y más aún, malos maestros, son el verdadero obstáculo para la deseada igualdad.

Del punto de vista de los alumnos, sostengo sistemáticamente, que lo que por lo general consideramos el fracaso de la educación, no es un fracaso en absoluto, sino más bien una estrategia de adaptación exitosa a los contextos socio-históricos hegemónicos occidentales. Afirmo que las perspectivas paradigmáticas occidentales han dado forma a la epistemología, la retórica y la práctica de la educación de tal manera, que ocultan el hecho que el "fracaso" de la educación no se debe a la calidad de las personas (profesores y / o estudiantes), sino a la de los sistemas, los mismos sistemas que, llamados liberales, conjuntamente y cooperativamente construyen y sostienen (en y con el mundo) para su propio bien (Varenne y McDermott, 1998).

Para no dejarlos en suspenso, quiero recordarles que mi apreciación del (así llamado) mundo liberal en el occidente, está influenciada y alineada con lo dicho por el gran antropólogo francés Loui Dummont, que apreciaba que los liberales occidentales, estando profundamente comprometidos con la igualdad, al encontrarse con alguien que no es igual (a ellos, pues este es el verdadero sentido de la igualdad occidental liberal), no tienen más remedio que matarlo (Dumont, 1986).

Al sugerirles que revisemos críticamente nuestras fuentes paradigmáticas, lo que verdaderamente deberíamos preguntarnos es: “¿Cómo se organiza el mundo y sus comunidades, de tal manera que les permita o sea valioso tener éxito en la educación, mientras que se le llama a éste éxito, fracaso? En resumen, la propuesta es que si queremos ser serios sobre la educación, debemos abandonar las perspectivas románticas, y con éstas, cambiar nuestros intereses. Nuestro interés 
no debe estar en los individuos del sistema, los alumnos o maestros, y menos aún en sus mentes. Debemos transferir nuestro interés de los individuos a los sistemas y sus políticas.

Tal vez, otra forma de preguntarse esto mismo sería “¿Cómo el mundo y las comunidades se organizan para que valga la pena hacer que sus esfuerzos educativos fracasen?" En pocas palabras, si estamos interesados en la reforma educacional, debemos cambiar el foco de nuestro interés de las mentes individuales de los estudiantes o los maestros, a los sistemas sociales y sus políticas.

Los alumnos que fracasan, no carecen de motivación ni de inteligencia, todos los hombres son real y naturalmente iguales; los que fracasan, por lo general, son los marginados, ya que al fracasar tienen realmente éxito. La pregunta es: ¿a qué?, ¿en qué tienen éxito? Los fracasados (los pobres y marginados) son exitosos en adaptarse a un sistema que deja poco lugar para los exitosos.

Los "dominios de éxito" de la cultura occidental son por lo general, de un modo u otro, homogéneos, y por lo tanto muy occidentales. Por lo que las personas interesadas en la educación liberal harían bien en pasar del estudio de los niños y maestros (fracasando cada uno en el colegio o fracasando frente al otro), al estudio de contextos culturales en los que el fracaso hace sentido. Cuando hagamos esto, estaremos confrontados con la política; el tema es que la educación bajo el dominio nacional liberal dice que la política no debe ser parte de la educación. En otras palabras: nos encontraremos hablando sobre aquello que el soberano prefiere que no hablemos. Al ser así, estaremos en peligro... el peligro es bueno para la educación... de todos? No! sólo para la educación de aquellos que ya no tienen nada que perder, pues el único éxito que se les ha permitido tener, es el fracaso.

El eje central del éxito de la educación formal nacional es su estructura y su funcionalidad, ambas basadas en expresiones de una perspectiva paradigmática particular, que dudamos puede ser beneficiosa. Las escuelas son el conducto central para la transmisión de dos creencias interrelacionadas del mundo occidental moderno: la primera, la creencia en el ser individual; y la segunda, que este individuo puede absorber conocimientos si está bien guiado.

Estos elementos mencionados anteriormente han sido creados a través de siglos de actividad humana en el funcionamiento de las escuelas. Más de 5000 años atrás, cuando las primeras escuelas fueron fundadas con el fin de producir un molde de escribas capaces de sostener las necesidades burocráticas y de crecimiento de los nuevos y poderosos centros urbanos centralizados en la Mesopotamia, ya estaban desarrolladas las tres características centrales que se mantienen hasta nuestros días (Cole, 1990; Goody, 1987):

- El estudiante es educado por extraños, separado de su familia y amigos;

- El conocimiento programado para la transmisión es diferenciado y compartimentado en campos de especialización; y

- El aprendizaje tenía lugar fuera del contexto de su aplicación prevista, es decir estudiantes ensayaban el conocimiento 'fuera de contexto'. 
Deberíamos preguntar si: ¿puede un marco que tiene como premisa el distanciamiento de la persona del núcleo familiar y comunitario, servir a las personas que deben a su vez servir a la sociedad? ¿Puede una estructura que concibe e imparte conocimientos diferenciados y compartimentados (historia, física, educación cívica, etc.), servir al cultivo de seres humanos en el mundo que es un todo coherente y unido? Y, por último, ¿es factible esperar que los estudiantes, estudiando en el marco escolar que abstrae el saber de la actividad diaria, que lo mentaliza y no lo actualiza, aprendan algo relevante en relación al hacer del mundo?

Estos son los problemas (o las soluciones) comunes de la educación formal, que se desarrolla separada en el tiempo y el espacio.

Los valores universales y el individualismo, son más dos elementos paradigmáticos del occidente que, si se dejan sin tratar, no nos permitirán que el sistema sea reformado y por lo tanto, que sea beneficioso para aquellos que deseen mantener un nivel de independencia con el fin de participar en el trabajo interpretativo y configurativo del mundo que habitan. Estas características paradigmáticas, a las que he hecho alusión anteriormente, son lo que la modernidad llama "valores universales" (es decir conceptualizaciones localizadas y restringidas del proceso occidental de colonización) y sus beneficiarios designados, "individuos autónomos" (que es también un concepto restringido y occidental), con sus identidades (otra palabra difícil de entender pero muy occidental) (Bekerman, 2000).

Este no es el lugar para exponer una plena crítica de estas perspectivas paradigmáticas de base occidental, será suficiente decir que tanto la cultura, como un molde identificable y reificado de comportamientos y creencias, y el individuo como autónomo y universal, han sido el foco de una controversia teórica de larga trayectoria en la alta y post modernidad. Esta controversia ha demostrado, me parece que con éxito, la relación entre estas categorías y muchas de las enfermedades actuales del mundo occidental (Giddens, 1991; Sampson, 1993; Taylor, 1994).

Vale la pena mencionar que estos desarrollos teóricos han señalado, entre otras cosas, dos temas centrales relacionados con nuestra comprensión actual de la cultura y la identidad individual que son relevantes para la educación. El primero, es que la cultura debe entenderse como un verbo, no un sustantivo, como algo que crece, que evoluciona de forma intermitente cuando es ejecutado, para disolverse rápidamente en la actividad continua del ser humano, actividad que puede reproducir o no de nuevo esa actividad, así llamada cultural, en forma similar o diferente en la próxima instancia de actividad humana (Bauman, 1999). En segundo lugar, la identidad individual debe ser concebida en forma similar, como un verbo, un proceso dialógico del devenir y de la formación, sobre todo a través del uso de la más humana de las herramientas humanas: el lenguaje (Harre y Gillett, 1995; Holland y Lave, 2001).

Así, la cultura y la identidad individual han llegado a ser conceptualizadas como evolucionarias, en procesos que dependen ampliamente de lenguaje (Maturana y Varela, 1987; Wittgenstein, 1953). Es dudoso que, no exponer estos paradigmas dominantes del individuo cosificado en la 
identidad y la cultura, junto con la no exposición de las prácticas a través de las cuales estos paradigmas son construidos e implementados dentro del sistema escolar, sea útil si lo que queremos es reformar la educación. El individuo y su identidad, decontextualizado e aislado, puede ser un excelente candidato para ser dominado, pero no es valioso si lo que queremos es el cambio social. Una cultura cosificada y segregada, puede ser una buena receta para ofrecer un barato reconocimiento multicultural a minorías marginadas, pero sirve más aún para justificar y perpetuar el continuo sufrimiento de estas mismas minorías, ahora reconocidas pero con su subordinación estructural intacta (Zvi Bekerman, 2002, 2004).

Permítanme concluir esta sección con un resumen de los principales puntos que he planteado, que son contrarios a la teoría y la práctica aceptada en la educación liberal.

1 - La percepción de fracaso, o insatisfacción con los productos de la educación, tienen poco que ver con la calidad de los maestros y estudiantes, y mucho que ver con la calidad de los sistemas que (todos) cooperativamente construimos.

2 - No apreciar el primer punto significa confundir el fracaso con actividades adaptadas a sistemas globales, locales y sus circunstancias.

3 - Perspectivas paradigmáticas positivistas occidentales son responsables por las actuales perspectivas educativas que guían a la teoría educativa liberal y su práctica. El cambio educativo sólo se producirá después que cambiemos estas perspectivas paradigmáticas.

4 - Cambiar las perspectivas paradigmáticas significa que la educación no puede ser más un proceso considerado como aislado en un individuo autónomo, situado en una cultura coherente estática y externa, y transmitida a través de las tareas específicas y medibles de los docentes. Cambiar las perspectivas paradigmáticas significa entender que el individuo, la cultura y el proceso de aprendizaje se representan mejor como contextualizados, historificados e interactivos.

5 - Esto implica que desde cualquier teoría de comunicación viable, para que la educación sea relevante, ésta tiene que ser relevante en y para el mundo, un punto casi trivial cuando se considera que su significado es por definición, no una representación dada, sino el resultado de la gestión de diferencia en una trayectoria histórica.

6 - Por último, he sugerido, que si lo anterior fuese correcto, haríamos bien en buscar mejores soluciones en la reorganización de la política actual del mundo occidental que en los parámetros limitados de sus centros escolares o en las mentes individuales de los maestros o estudiantes.

\section{Casi para finalizar}

Las investigaciones actuales muestran claramente que las reformas educativas implementadas en las sociedades occidentales durante el último medio siglo han logrado poco en todo lo que se refiere a mejorar las posibilidades de los indigentes (Anyon, 1995; Apple, 1999; Berliner, 2006; 
Hirschland y Steinmo, 2003; Ravitch, 2000; Sarason, 1990; Tyack y Cuban, 1995). La situación no cambia mucho cuando se consideran las reformas propuestas en el campo de la formación docente. Esto no debería ser una sorpresa, sobre todo al considerar las tareas que se le han pedido cumplir a la educación universal en el marco de los estados nacionales -ayudar a dar forma a la "conciencia" y la ideología nacional (Billig, 1995; Gellner, 1983).

Es la retórica nacional, a la que todos estamos acostumbrados, que pone en las escuelas y los profesores la responsabilidad de encontrar "soluciones" a los problemas que perturban a las sociedades modernas. Es en este hacer del hablar que la educación y el bien común se construyen retóricamente por los Estados-nación como objetivos necesarios y dignos, alcanzables a través de la educación, dentro de un marco cultural que ya se considera de apoyo de la igualdad y los ideales de tolerancia. Es precisamente esta construcción retórica que nos preocupa. Porque, al igual que el hecho que otras construcciones prometidas, como la reforma social y la igualdad no se ha logrado a través de la educación, se puede dudar que el bien común sea logrado a través de ella. El compromiso con la retórica de la reforma a través de la educación dentro de las estructuras del Estado-nación, por lo general implica abrazar un dilema en el que, si bien no está permitido decir que el cambio no está disponible, la educación es ofrecida en forma universal, libre y obligatoria, los contextos en los que la educación evoluciona, se establecen de tal manera que no se permite que la educación/escolarización produzca el cambio deseado.

Recordemos que toda medición educacional para propósitos de reforma, cada modelo y cada conjunto de datos, fue electo por un proceso político, que a la vez construyó ese modelo y definió el éxito para ese modelo, y para su propio bien — que no es necesariamente el bien de los alumnos.

No sólo las medidas son dictados políticos. Son peor, pues son aquellas cosas que es fácil medir en forma totalmente desconectada del hecho que si lo medido es valioso medirlo en términos del bien de la población.

Y, ¿qué medimos o decimos que medimos?, la efectividad del maestro, lo que pesamos que enseña, aunque no tenemos claras definiciones de qué es el saber (más que la 'correcta' respuesta a un examen). Medimos tiempo y alcance curricular, aunque no es clara la relación currícula-tiempo, como en los exámenes que bajo el pretexto de medir el saber, miden el tiempo para poder diferenciar entre los buenos alumnos (los que terminan rápido), y los malos, los idiotas y los inteligentes... a pesar de bien saber que no hay idiotas o inteligentes, sólo pobres y ricos.

¿Tal vez vale la pena medir otros parámetros?

Tal vez sería interesante medir el nivel de acceso a la universidad. En muchos de los países occidentales, la universidad tiene un costo que la gran mayoría de la población no puede pagar, y cuando la universidad es gratis, no es claro que los colegios de los destituidos y maginados han preparado a los estudiantes para pensar sobre la posibilidad de registrarse a estudiar en la universidad, y ni hablemos de haberlos preparado para las competencias necesarias para estos estudios universitarios. 
¿Acaso no sería interesante medir los niveles de pobreza infantil, de violencia en el vecindario, la falta de vivienda, y los familiares encarcelados de chicos que atienden escuelas en zonas marginales? ¿Acaso estos factores no tienen nada que ver con el éxito de la educación?

$\mathrm{Y}$, ¿qué hay con medir el número de embarazos no deseados?: las investigaciones realizadas en Europa han encontrado que los niños y adolescentes nacidos como resultado de embarazos no deseados, suelen recibir calificaciones menores en la temprana infancia y la adolescencia.

Sería también interesante medir la disponibilidad de la atención prenatal: la investigación ha encontrado que la atención prenatal (que es realmente para la madre pero a los hombres no les gusta decir esto) en la primera infancia tiene efectos sustanciales sobre los resultados académicos y cognitivos. Y que para sorpresa, los de clase media atienden más a sus hijos (o al menos así nos lo dicen) que los pobres...será porque los pobres simplemente no cuentan con el tiempo necesario?

Podemos medir mucho más, como por ejemplo el acceso a la educación preescolar, a las bibliotecas o a las computadoras.

Medir esta larga lista de parámetros contextualizaría la limitada perspectiva del fracaso... pero no es conveniente, porque al hacerlo nos obligaría a enfocarnos en la sociedad que el mundo moderno y occidental ha desarrollado y no nos permitiría culpar a los maestros, a los estudiantes (o sus familias) ni a las escuelas. Medir esta larga lista de parámetros obligaría al estado nacional y sus élites a preguntarse sobre su propia responsabilidad en el éxito de asegurar que 50\% de la población fracase en las escuelas.

Como ya hemos mencionado, los colegios enfatizan y glorifican el ritmo diferencial del aprender, a tal punto que el ritmo del aprender, más que lo aprendido, se vuelve el parámetro para medir quién es mejor. La verdad es, que la historia de la humanidad o la evolución y su desinteresado desarrollo, ha hecho que todos sepan mucho y que todos puedan saberlo todo. Esta es una simple verdad que surge de otra simple verdad: todos los humanos son iguales. Preste atención el lector que digo 'son' iguales y no digo 'tienen que ser'. La segunda opción ('tienen que ser' iguales) es la mentirosa promesa de las burocracias nacionales interesadas en mantener, a través de instituciones formales de aprendizaje (colegios), la desigualdad. La educación universal es presentada por el estado-nación como la entrega gratuita al pueblo del conductor hacia la igualdad, una igualdad que colegios en ninguna parte del mundo han podido realizar. La primera opción ('son' iguales) es la verdad empírica y dependiendo de la inclinación ideológica/teológica del lector la verdad.

La verdad es que el humano, desde el momento que nace, sólo aprende/sabe en el sentido mencionado. Tanto es así, que nos parece correcto decir que aprender/saber es sinónimo de vida. El aprender, el saber, no es una condición humana especial sino es la condición humana básica y única. Todos aprenden todo (lo que copian, hacen y practican) siempre. El aprender, el saber, es la respuesta de la vida, de la biología, a la necesidad. A todas las necesidades humanas. Necesidades físicas, materiales, simbólicas, etc. Se aprende sólo lo que se hace, y se es lo que uno hace también. Los filósofos tradicionales, de Platón hasta Descartes, son excelentes amigos de los poderosos. 
Las abstracciones que nos ofrecen, son un gran placebo para mantener a los poderosos en el poder. Ellos, como los maestros certificados por el Estado, quieren certificar nuestro saber, quieren legitimarlo en lugar que sea legitimado por la práctica. ¿Recuerdan el colegio? En éste, por lo general, había una sola forma correcta de llegar a una solución. Había una sola forma de entender un texto. Los maestros y filósofos pretendían saber lo que el texto decía, mejor que nuestra propia lectura sobre ese mismo texto. Parece que lo que querían, en vez de que entendiéramos el texto, era que lo hiciéramos como ellos lo entendían. Y para conseguir que nuestro entendimiento fuera el de ellos, se apoyaban en lo efímero (sus palabras en la oralidad), más que en lo material, en lo empírico de la escritura del texto dado a leer. Los amigos del saber como práctica, son amigos de la igualdad. Los amigos del saber, saben que el saber se legitima en las comunidades en las cuales se aplica y que se gana a través del copiar, del repetir, del comparar, del hacer y del practicar (Ranciere, 1991).

No cabe duda que todos los que fracasan, y los que no, somos copartícipes en la producción del fracaso y el éxito, la pareja inseparable que se necesita uno al otro para vivir. Los que alcanzan el éxito, no necesitan de nuestra ayuda, los que alcanzan el fracaso, deben saber que éste conlleva el mismo trabajo que alcanzar el éxito. También el fracaso es aprendido en la práctica, y sólo a través de la práctica se puede cambiar. Todos pueden saber todo, pero no en las presentes circunstancias. Avanzar el saber tiene más que ver con cambiar las circunstancias en las cuales el aprender/saber se produce, que con cambiar a los individuos, que son los supuestos portadores del aprender/saber. Dicen que el gran pragmatista George Herbert Mead decía que los expertos son malos para la democracia. Me parece que tenía razón. Pongan un experto en escena y muchas voces se apagarán. El aprender/saber tiene que ver más con los contextos que construimos para que nuestros alumnos habiten, que con las cualidades de las mentes que no tienen, o con los currículos que les escribimos.

\section{Bibliografía}

Anyon, Jean (1995). "Race, social class, and educational reform in an inner-city school". Teachers College Record, 97(1), 69-94.

Apple, Michael W. (1999). "Rhetorical reforms: Markets, standards and inequality". Current Issues in Comparative Education, 1(2), 6-18.

Barrett, P. H., P. J.Gautrey, S. Herbert, D. Kohn y S. Smith (eds.) (1987). Charles Darwin's notebooks, 1836-1844: Geology, transmutation of species, metaphysical enquiries. Ithaca, New York: Cornell University Press and British Museum (Natural History); London: Cambridge University Press and British Museum (Natural History).

Bateson, G. (1979). Mind and nature a necessary unity. New York: Bantam Books.

Bauman, Z. (1999). Culture as praxis. London: Sage.

Beck, Ulrich (2011). "Cosmopolitanism as imagined communities of global risk". American Behavioral Scientist, 55(10), 1346-1361. 
Bekerman, Z. (2006). "It's We, the Researchers, Who are in Need of Renovation". Journal of Research Practice, 2(1), Article, p. 1.

_ (2000). "Dialogic directions: Conflicts in Israeli/Palestinian education for peace". Intercultural Education, 11(1), 41-51.

_ (2002). "Hidden Dangers in Multicultural Discourse". Race Equality Teaching, 21(3), 36-41. (2004). "Multicultural approaches and options in conflict ridden areas: Bilingual PalestinianJewish education in Israel". Teachers College Record, 106(3), 574-610.

Berliner, David C. (2006). Our impoverished view of educational reform. Teachers college Record, 108(6), 949-995.

Billig, Michael. (1995). Banal nationalism. London: Sage.

Burke, Kenneth (1969). A rhetoric of motives. Berkeley: University of California Press.

— (1969). A grammar of motives. Berkeley: University of California Press.

Churchman, C. W. (1968). Challange to reason. New York: Mc Graw-Hill. (1979). The systems approach (2nd ed.). New York: Dell Publishing.

Cole, Michael. (1990). Cultural psychology: A once and future discipline? En J. J. Berman (ed.). Nebraska symposium on motivation, 1989: Cross-cultural perspectives (vol. 37, pp. 179-335). Lincoln: University of Nebraska Press.

— (2001). "Remembering history in sociocultural research". Human Development, 44(2/3), 166-169.

Conant, J. B. (1951). Science and Common Sense. New Haven: Yale University Press.

Derrida, J. (1987). The Truth in Painting (G. B. a. I. McLeod, Trans.). Chicago, IL: Chicago University Press.

Dumont, Louis. (1986). “Are Cultures Living Beings? German Identity in Interaction”. Man, 21(4), 587-604.

Duro, P. (1996). The Rhetoric of the Frame Cambridge: Cambridge University Press.

Foucault, M. (1969). The archeology of knowledge. London: Tavistock.

— (1973). Madness \& Civilization (R. Howard, Trans.). New York: Vintage Books.

Geertz, C. (1973). The interpretation of cultures. New York: Basic Books.

Gellner, E. (1983). Nations and nationalism. Oxford: Basic Blackwell.

Giddens, A. (1991). Modernity and self identity. Stanford: Stanford University Press.

Goody, J. (1987). The interface between the written and the oral. Cambridge: Cambridge University Press.

Harre, R. y G. Gillett (1995). The discoursive mind. London: Sage.

Hirschland, Matthew J. y Sven Steinmo (2003). "Correcting the Record: Understanding the History of Federal Intervention and Failure in Securing U.S. Educational Reform". Educational Policy, 17(343-364).

Holland, Dorothy y Jean Lave (eds.). (2001). History in Person. Santa Fe and Oxford: School of American Research Press \& James Currey. 
Maturana, Humberto y F. Varela (1987). The tree of knowledge. Boston: Shambhala. Mayr, Ernst. (1988). Toward a new philosophy of biology. Cambridge: Belknap Harvard.

Neuman, Yair y Zvi Bekerman (2001). "Cultural resources and the gap between educational theory and practice". Teachers College Record, 103(3), 471-484.

Ortega y Gasset, José (1957). Man and people. New York: Norton.

Ranciere, J. (1991). The ignorant schoolmaster: Five lessons in intelectual emancipation (K. Ross, Trans.). Stanford: Stanford University Press.

Ravitch, Diane (2000). Left Back: A Century of Failed School Reforms. New York: Simon \& Schuster.

Sampson, E. E. (1993). Celebrating the other: A dialogic account of human nature. Hertfordshire: Harvester Wheatsheaf.

Sapir, E. (1921). Language. Harcourt: Bace \& Co.

Sarason, S. (1990). The predictable failure of educational reform. San Francisco: Jossey-Bass.

Taylor, Charles. (1994). "The politics of recognition”. In D. T. Goldberg (ed.), Multiculturalism: A critical reader (pp. 75-106). Oxford: Blackwell.

Tyack, David y Larry Cuban (1995). Tinkering toward Utopia: A Century of Public School Reform. Cambridge: Harvard University Press.

Varenne, H.y R. McDermott (1998). Successful Failure: The Schools America Builds. Colorado: Westview Press.

Wittgenstein, Ludwig (1953). Philosophical investigations (G. E. M. Anscombe, Trans.). Oxford: Blackwell.

Recibido: 02/05/14

Dictaminado: 24/09/14

Corregido: 06/10/14

Aceptado: 07/11/14 